A. G. Nikitin (Inst. Math. Nat. Acad. Sci. Ukraine, Kyiv)

\title{
GROUP CLASSIFICATION OF SYSTEMS \\ OF NONLINEAR REACTION-DIFFUSION EQUATIONS WITH TRIANGULAR DIFFUSION MATRIX
}

\section{ГРУПОВА КЛАСИФІКАЦІЯ СИСТЕМ НЕЛІНІЙНИХ РІВНЯНЬ РЕАКЦЇ̈-ДИФУЗІї З ТРИКУТНОЮ МАТРИЦЕЮ ДИФУЗІї}

Group classification of systems of two coupled nonlinear reaction-diffusion equations with a general diffusion matrix started in previous author's works completed in present paper where all nonequivalent equations with triangular diffusion matrix are classified. In addition, symmetries of diffusion systems with nilpotent diffusion matrix and additional first order derivative terms are described.

Завершено групову класифікацію систем двох зачеплених рівнянь реакції-дифузії з загальною матрицею дифузії, розпочату в попередніх роботах автора. А саме, прокласифіковано всі нееквівалентні рівняння $з$ трикутною матрицею дифузії. Крім цього, описано симетрії дифузійних систем з нільпотентною матрицею дифузії та додатковими членами з похідними першого порядку.

1. Introduction. Group classification is a corner stone of qualitative analysis of differential equations. Such classification makes it possible to specify equivalence relations in a considered class of equations. In addition, it opens a way to application of powerful group-theoretical tools for searching for conservation laws, construction of exact solutions, group generation of families of solutions starting with known ones, etc.

In the present paper we complete the group classification of systems of reactiondiffusion equations with general diffusion matrix performed in papers $[1,2]$. These equations can be written in the following form:

$$
\begin{aligned}
& u_{t}-\Delta_{m}\left(A^{11} u+A^{12} v\right)=f^{1}(u, v), \\
& v_{t}-\Delta_{m}\left(A^{21} u+A^{22} v\right)=f^{2}(u, v),
\end{aligned}
$$

where $u$ and $v$ are function of $t, x_{1}, x_{2}, \ldots, x_{m}, A^{11}, A^{12}, A^{21}$ and $A^{22}$ are elements of diffusion matrix (which are supposed to be constant) and $\Delta_{m}$ is the Laplace operator in $R^{m}$.

Equations (1) form the grounds for a great many models of mathematical physics, biology, chemistry, etc., which makes their group classification to be especially relevant. A survey of investigation of symmetries of systems (1) can be found in $[1,2]$.

Up to linear transformation of dependent variables there exist three a priori nonequivalent versions of equations (1), corresponding to a diagonal, triangular and square diffusion matrix. The equations with diagonal and square diffusion matrix have been classified in papers [1] and [2] correspondingly.

Here we consider the remaining case when the diffusion matrix is triangular and system (1) is reduced to the following form:

$$
\begin{gathered}
u_{t}-a \Delta_{m} u=f^{1}(u, v), \\
v_{t}-\Delta_{m} u-a \Delta_{m} v=f^{2}(u, v),
\end{gathered}
$$

where $a$ is a real constant. 
In the case when $a=0$ the diffusion matrix is nilpotent and it is naturally to generalize (2) to the following system:

$$
\begin{gathered}
u_{t}-p_{\mu} v_{x_{\mu}}=f^{1}(u, v), \\
v_{t}-\Delta u=f^{2}(u, v),
\end{gathered}
$$

were summation from 1 to $m$ is imposed over the repeated indices, $p_{\mu}$ are arbitrary constants. Up to linear transformations of independent variables

$$
p_{1}=p_{2}=\ldots=p_{m-1}=0, \quad p_{m}=p,
$$

where $p=\sqrt{p_{1}^{2}+p_{2}^{2}+\ldots+p_{m}^{2}}$.

We notice that in addition to applications in mathematical biology equations (3) can serve as a potential ones for the nonlinear D'Alembert equation.

2. Symmetries and determining equations. To describe symmetries of equations (2) and (3) with respect to continuous groups of transformations we use the Lie infinitesimal approach. Using the standard Lie algorithm we find the determining equations for coordinates $\eta, \xi_{a}, \pi^{b}$ of generator $X$ of the symmetry group:

$$
X=\eta \partial_{t}+\xi^{v} \partial_{x_{v}}-\pi^{1} \partial_{u}-\pi^{2} \partial_{v}
$$

The first set of determining equations describes dependence of $\eta, \xi^{v}$ and $\pi^{b}$ on $u$ and $v$ :

$$
\eta_{u}=\eta_{v}=0, \quad \xi_{u}^{v}=\xi_{v}^{v}=0, \quad \pi_{u u}^{a}=\pi_{u v}^{a}=\pi_{v v}^{a}=0 .
$$

Here and in the following subscripts denote derivatives with respect to the corresponding variables, i.e., $\eta_{v}=\frac{\partial \eta}{\partial v}$, etc.

In accordance with (6) $\eta$ and $\xi^{\nu}$ are functions of $t$ and $x_{\mu}$ only, and

$$
\pi^{a}=N^{a 1} u+N^{a 2} v+B^{a}, \quad a=1,2,
$$

where $N^{a b}, B^{a}$ are functions of $t$ and $x_{\mathrm{v}}$. The remaining determining equations in the case $a \neq 0$ are [1]:

$$
\begin{gathered}
2 A \xi_{x_{\mu}}^{v}=-\delta_{m}^{\mu v}\left(\eta_{t} A+[A, N]\right), \quad \eta_{x_{v} t}=0 \\
\xi_{t}^{v}-2 a N_{x_{v}}^{11}-a \Delta_{m} \xi^{v}=0, \quad N^{12}=0, \quad N_{x_{v}}^{21}=-a N_{x_{v}}^{11} \\
\eta_{t} f^{k}+N^{k b} f^{b}+\left(N_{t}^{k b}-\Delta_{m} A^{k s} N^{s b}\right) u_{b}+B_{t}^{k}-\Delta_{m} A^{k c} B^{c}=\left(B^{a}+N^{a b} u_{b}\right) f_{u_{a}}^{k} .
\end{gathered}
$$

Here $N$ and $A$ are matrices whose elements are $N^{a b}$ and $A^{a b}, \delta^{a b}$ is the Kronecker symbol, and we use the temporary notation $u=u_{1}, v=u_{2}$.

Using (7) - (10) we find the general form of symmetry (5):

$$
\begin{gathered}
X=\Psi^{\mu v} x_{\mu} \partial_{x_{v}}+v \partial_{t}+\rho_{\mu} \partial_{x_{\mu}}+\lambda K+\sigma_{\mu} G_{\mu}+\omega_{\mu} \hat{G}_{\mu}+\mu D- \\
-C^{1}\left(u \partial_{u}+v \partial_{v}\right)-C^{2} u \partial_{v}-B^{1} \partial_{u}-B^{2} \partial_{v}
\end{gathered}
$$

where the Greek letters denote arbitrary constants, $B^{1}, B^{2}$ are functions of $t, x$, and $C^{1}, C^{2}$ are functions of $t$, 


$$
\begin{gathered}
K=2 t\left(t \partial_{t}+x_{\mu} \partial_{x_{\mu}}\right)-\frac{x^{2}}{2}\left(\frac{1}{a}\left(u \partial_{u}+v \partial_{v}\right)-u \partial_{v}\right)-t m\left(u \partial_{u}+v \partial_{v}\right), \\
G_{\mu}=t \partial_{x_{\mu}}+\frac{1}{2} x_{\mu}\left(\frac{1}{a}\left(u \partial_{u}+v \partial_{v}\right)-\frac{1}{a^{2}} u \partial_{v}\right), \\
\hat{G}_{\mu}=e^{\gamma t}\left(\partial_{x_{\mu}}+\frac{1}{2} \gamma x_{\mu}\left(\frac{1}{a}\left(u \partial_{u}+v \partial_{v}\right)-\frac{1}{a^{2}} u \partial_{v}\right)\right), \\
D=t \partial_{t}+\frac{1}{2} x_{\mu} \partial_{x_{\mu}} .
\end{gathered}
$$

For $a=0$ symmetry $X$ again has the form (11) where however $\lambda=\sigma_{\mu}=\omega_{\mu}=0$. In addition, $B^{2}$ can depend not on $t, x$ only, but also on $u$.

In accordance with (5), (7) and (10) equation (2) admits symmetry (11) iff the following classifying equations are satisfied:

$$
\begin{gathered}
\left(\lambda(m+4) t+\mu+\frac{1}{a}\left(\frac{1}{2} \lambda x^{2}+\sigma_{\mu} x_{\mu}+\gamma e^{\gamma t} \omega_{\mu} x_{\mu}\right)+C^{1}\right) f^{1}+ \\
+C_{t}^{1} u+B_{t}^{1}-a \Delta B^{1}=\left(B^{1} \partial_{u}+B^{2} \partial_{v}+C^{1}\left(u \partial_{u}+v \partial_{v}\right)+C^{2} u \partial_{v}+\right. \\
\left.+\lambda m t\left(u \partial_{u}+v \partial_{v}\right)+\left(\frac{1}{2} \lambda x^{2}+\sigma_{\mu} x_{\mu}+\gamma e^{\gamma t} \omega_{\mu} x_{\mu}\right)\left(\frac{1}{a}\left(u \partial_{u}+v \partial_{v}\right)-\frac{1}{a^{2}} u \partial_{v}\right)\right) f^{1}, \\
\left(\lambda(m+4) t+\mu+C^{1}\right) f^{2}+C^{2} f^{1}+\left(\frac{1}{2} \lambda x^{2}+\sigma_{\mu} x_{\mu}+\gamma e^{\gamma t} \omega_{\mu} x_{\mu}\right)\left(\frac{1}{a} f^{2}-f^{1}\right)+ \\
+C_{t}^{1} v+C_{t}^{2} u+B_{t}^{2}-\Delta B^{2}-a \Delta B^{1}=\left(B^{1} \partial_{u}+B^{2} \partial_{v}+C^{1}\left(u \partial_{u}+v \partial_{v}\right)+C^{2} u \partial_{v}+\right. \\
\left.+\lambda m t\left(u \partial_{u}+v \partial_{v}\right)+\left(\frac{1}{2} \lambda x^{2}+\sigma_{\mu} x_{\mu}+\gamma e^{\gamma t} \omega_{\mu} x_{\mu}\right)\left(\frac{1}{a}\left(u \partial_{u}+v \partial_{v}\right)-\frac{1}{a^{2}} u \partial_{v}\right)\right) f^{2} .
\end{gathered}
$$

Equations (13) are nothing but a special case of the generic classifying equations (15), ref. [1] which are valid for arbitrary invertible diffusion matrix.

Equation (3) needs a particular analysis. For $p \neq 0$ the related symmetry operator (5) reduces to the form

$$
X=X_{0}+\tilde{X}
$$

where

$$
\begin{gathered}
X_{0}=\lambda \partial_{t}+v_{\alpha} \partial_{x_{\alpha}}+\Psi^{k l} x_{k} \partial_{l}, \\
\tilde{X}=\mu\left(3 t \partial_{t}+2 x_{v} \partial_{v}-v \partial_{v}\right)-F\left(u \partial_{u}+v \partial_{v}\right)-B^{1} \partial_{u}-B^{2} \partial_{v} .
\end{gathered}
$$

Here $\Psi^{\mu \nu}$ is an antisymmetric tensor and summation is imposed over the repeated Greek and Latin indices from 1 to $m$ and from 1 to $m-1$ correspondingly.

Symmetries $X_{0}$ are admitted by equations (3), (4) with arbitrary nonlinearities $f^{1}$ and $f^{2}$ while the classifying equations generated by symmetries $\tilde{X}$ are

$$
\begin{aligned}
& (3 \mu+F) f^{1}+F_{t} u+B_{t}^{1}-p B_{x_{m}}^{2}=\left(B^{1} \partial_{u}+B^{2} \partial_{v}+F u \partial_{u}+(F+\mu) v \partial_{v}\right) f^{1}, \\
& (4 \mu+F) f^{2}+F_{t} v+B_{t}^{2}-\Delta B^{1}=\left(B^{1} \partial_{u}+B^{2} \partial_{v}+F u \partial_{u}+(F+\mu) v \partial_{v}\right) f^{2},
\end{aligned}
$$

where $F$ and $B^{1}, B^{2}$ are unknown functions of $t$ and $t, x$ respectively. 
The determining equations for symmetries of equation (3) with $p=0$ are qualitatively different for the cases, when the number $m$ of spatial variables $x_{1}$, $x_{2}, \ldots, x_{m}$ is $m=1, m=2$ and $m>2$. The related generator (5) has the form

$$
\begin{gathered}
X=\alpha D+\left(\int(N-M) d t\right) \partial_{t}+2 m H^{\alpha} \partial_{x_{a}}-\left(N+(m-2) H_{x_{a}}^{a}\right) u \partial_{u}- \\
-\left(M+(m+2) H_{x_{\alpha}}^{\alpha}\right) v \partial_{v}-B^{1} \partial_{u}-B^{2} \partial_{v}-B^{3} u \partial_{v},
\end{gathered}
$$

where summation from 1 to $m$ is imposed over repeating indices, the Greek letters denote arbitrary parameters, $M, N$ are functions of $t, B^{1}, B^{3}$ are functions of $t, x$, $B^{2}$ are functions of $t, x, u$, and

$$
H^{a}=2 \lambda_{b} x_{b} x_{a}-x^{2} \lambda_{a} \text { if } m>2 .
$$

For $m=2 H^{a}$ are arbitrary functions satisfying the Cauchy - Riemann conditions:

$$
H_{x_{1}}^{1}=H_{x_{2}}^{2}, \quad H_{x_{2}}^{1}=-H_{x_{1}}^{2} .
$$

In the case when $m=1 \quad H^{1}$ is a function of $x$ and the sums with respect to $a$ in (17) are reduced to single terms.

The related classifying equations have the form

$$
\begin{gathered}
\left(\alpha+2 N-M+(m-2) H_{x_{a}}^{a}\right) f^{1}+N_{t} u+B_{t}^{1}=\left(B^{1} \partial_{u}+B^{2} \partial_{v}+\right. \\
\left.+B^{3} u \partial_{v}+\left(N+(m-2) H_{x_{a}}^{a}\right) u \partial_{u}+\left(M+(m+2) H_{x_{a}}^{a}\right) v \partial_{v}\right) f^{1} \\
\left(\alpha+N+(m+2) H_{x_{a}}^{a}\right) f^{2}+B^{3} f^{1}+M_{t} v+B_{t}^{3} u+B_{t}^{2}-\Delta B_{1}+ \\
+(2-m) \Delta H_{x_{a}}^{a} u=\left(B^{1} \partial_{u}+B^{2} \partial_{v}+B^{3} u \partial_{v}+\left(N+(m-2) H_{x_{a}}^{a}\right) u \partial_{u}+\right. \\
\left.+\left(M+(m+2) H_{x_{a}}^{a}\right) v \partial_{v}\right) f^{2} .
\end{gathered}
$$

We notice that in this case symmetry classification appears to be rather complicated and cumbersome. Nevertheless, the classifying equations can be effectively solved using the approach outlined in the following sections.

3. Classification of symmetries. Following [1] we specify basic, main and extended symmetries for the analyzed systems of reaction-diffusion equations.

Basic symmetries form the kernel of the main symmetry group and so are admitted by equation (2) with arbitrary nonlinearities $f^{1}$ and $f^{2}$. They are generated by the following infinitesimal operators:

$$
P_{0}=\partial_{t}, \quad P_{\lambda}=\partial_{x_{\lambda}}, \quad J_{\mu v}=x_{\mu} \partial_{x_{v}}-x_{v} \partial_{x_{\mu}}
$$

and correspond to shifts of independent variables and rotations of variables $x_{\mathrm{v}}$.

Main symmetries $\tilde{X}$ form an important subclass of general symmetries (11) which correspond to $\lambda=\sigma_{v}=\omega_{v}=0$ and have the following form:

$$
\tilde{X}=\mu D+Y, \quad Y=-C^{1}\left(u \partial_{u}+v \partial_{v}\right)-C^{2} u \partial_{v}-B^{1} \partial_{u}-B^{2} \partial_{v} .
$$

To describe all Lie symmetries admitted by equation (2) we follow the procedure outlined in [1] which includes the following steps:

Finding all main symmetries (20), i.e., solving equations (13) for $\Psi^{\mu \nu}=v=p_{v}=$ $=\sigma_{v}=\omega_{v}=0$ : 


$$
\begin{gathered}
\left(\mu+C^{1}\right) f^{1}+C_{t}^{1} u+B_{t}^{1}-a \Delta B^{1}=\left(C^{1}\left(u \partial_{u}+v \partial_{v}\right)+C^{2} u \partial_{v}+B^{1} \partial_{u}+B^{2} \partial_{v}\right) f^{1}, \\
\left(\mu+C^{1}\right) f^{2}+C_{t}^{2} u+C_{t}^{1} v+B_{t}^{2}-a \Delta B^{2}-\Delta B^{1}= \\
=\left(C^{1}\left(u \partial_{u}+v \partial_{v}\right)+C^{2} u \partial_{v}+B^{1} \partial_{u}+B^{2} \partial_{v}\right) f^{2} .
\end{gathered}
$$

Specifying all cases when the main symmetries can be extended, i.e., at least one of the following systems is satisfied:

$$
\begin{gathered}
a f^{1}=\left(a\left(u \partial_{u}+v \partial_{v}\right)-u \partial_{v}\right) f^{1}, \\
a f^{2}-f^{1}=\left(a\left(u \partial_{u}+v \partial_{v}\right)-u \partial_{v}\right) f^{2} ; \\
a\left(f^{1}+\gamma u\right)=\left(a\left(u \partial_{u}+v \partial_{v}\right)-u \partial_{v}\right) f^{1}, \\
a\left(f^{2}+\gamma v\right)-\gamma u=\left(a\left(u \partial_{u}+v \partial_{v}\right)-u \partial_{v}\right) f^{2}
\end{gathered}
$$

or if equation (22) is satisfied together with the following condition:

$$
(m+4) f^{a}=m\left(u \partial_{u}+v \partial_{v}\right) f^{a}, \quad a=1,2 .
$$

If relations (22), (23) or (24) are valid then the system (2) admits symmetry $G_{a}, \hat{G}_{\alpha}$ or $K$ correspondingly.

When classifying equations (3) or (2) with $a=0$ the second step in not needed in as much as in accordance with (17) and (15) these equations admit basic and main symmetries only.

The first step of the described procedure is rather complicated. In the next section we present specific tools to solve equations (21).

4. Algebras of main symmetries for equation (2). In accordance with the plane outlined in Section 3, to make symmetry classification of equations (2) we first describe the main symmetries generated by operators (20) and then indicate extensions of these symmetries.

First we note that for any $f^{1}$ and $f^{2}$ equation (2) admits the following equivalence transformations

$$
\begin{gathered}
u \rightarrow K^{1} u+b^{1}, \quad v \rightarrow K^{1} v+K^{2} u+b^{2}, \\
f^{1} \rightarrow \lambda^{2} K^{1} f^{1}, \quad f^{2} \rightarrow \lambda^{2}\left(K^{1} f^{2}+K^{2} f^{1}\right), \\
t \rightarrow \lambda^{-2} t, \quad x_{b} \rightarrow \lambda^{-1} x_{b},
\end{gathered}
$$

where $K^{1}, K^{2}, \lambda, b^{1}$ and $b^{2}$ are constants, $\lambda K_{1} K_{2} \neq 0$. Equivalence transformations keep the general form of equation (2) but can change the concrete realization of its right-hand side. For some nonlinearities $f^{1}$ and $f^{2}$ there exist additional equivalence transformation which will be specified in the following.

To solve rather complicated classifying equations (21), we use the main algebraic property of the main symmetries, i.e., the fact that they should form a Lie algebra (which we denote by $\mathcal{A}$ ). In other words, instead of going throw all nonequivalent possibilities arising via separation of variables in the classifying equations we first specify all nonequivalent realizations of algebra $\mathcal{A}$ for our equations up to arbitrary constants and arbitrary functions. Then we easily solve classifying equations (21) with known functions $C^{k}$ and $B^{a}$.

Consider consequently one-, two-, $\ldots, n$-dimensional algebras of operators (20). Let $\tilde{X}$ be a basis element of a one-dimensional algebra $\mathcal{A}$ then commutators of $\tilde{X}$ 
with $P_{0}$ and $P_{a}$ are equal to a linear combination of $\tilde{X}$ and operators (19). This can happen in the following cases:

$$
\begin{gathered}
C^{a}=\mu^{a}, \quad B^{a}=v^{a}, \\
C^{a}=e^{\lambda t} \mu^{a}, \quad B^{a}=e^{\lambda t} v^{a}, \\
C^{a}=0, \quad B^{a}=e^{\lambda t+\omega \cdot x} v^{a}, \quad \mu=0,
\end{gathered}
$$

where $\mu^{a b}, \mu^{a}, \lambda$, and $\omega=\left(\omega_{1}, \omega_{2}, \ldots, \omega_{m}\right)$ are constants, $\omega \cdot x=\omega_{v} x_{v}$ and $\omega \neq 0$.

To classify all nonequivalent symmetries (20), (26) we use isomorphism of the related operators $Y$ with $3 \times 3$ matrices of the following form:

$$
g=\left(\begin{array}{ccc}
0 & 0 & 0 \\
v^{1} & \mu^{1} & 0 \\
v^{2} & \mu^{2} & \mu^{1}
\end{array}\right)
$$

Equivalence transformations (25) generate the following transformation for matrix (27):

$$
g \rightarrow g^{\prime}=U g U^{-1}
$$

where

$$
U=\left(\begin{array}{ccc}
1 & 0 & 0 \\
b^{1} & K^{1} & 0 \\
b^{2} & K^{2} & K^{1}
\end{array}\right), \quad U^{-1}=\frac{1}{K^{1}}\left(\begin{array}{ccc}
K^{1} & 0 & 0 \\
-b^{1} & 1 & 0 \\
b^{1} K^{2}-b^{2} & -\frac{K^{2}}{K^{1}} & 1
\end{array}\right)
$$

Up to transformations (28) there exist six nonequivalent matrices $g$, i.e.,

$$
\begin{aligned}
& g_{1}=\left(\begin{array}{lll}
0 & 0 & 0 \\
0 & 1 & 0 \\
0 & 0 & 1
\end{array}\right), \quad g_{2}=\left(\begin{array}{lll}
0 & 0 & 0 \\
\lambda & 0 & 0 \\
1 & 0 & 0
\end{array}\right), \quad g_{3}=\left(\begin{array}{lll}
0 & 0 & 0 \\
1 & 0 & 0 \\
0 & 0 & 0
\end{array}\right) \text {, } \\
& g_{4}=\left(\begin{array}{lll}
0 & 0 & 0 \\
0 & 1 & 0 \\
0 & 1 & 1
\end{array}\right), \quad g_{5}=\left(\begin{array}{lll}
0 & 0 & 0 \\
0 & 0 & 0 \\
0 & 1 & 0
\end{array}\right), \quad g_{6}=\left(\begin{array}{lll}
0 & 0 & 0 \\
1 & 0 & 0 \\
0 & 1 & 0
\end{array}\right) \text {. }
\end{aligned}
$$

Let us denote

$$
\hat{g}=g^{22} u \partial_{u}+g^{33} v \partial_{v}+g^{32} u \partial_{v}+g^{21} \partial_{u}+g^{31} \partial_{v},
$$

where $g^{k s}$ are elements of matrix $g$. Then in accordance with (26) the related symmetries (20) have the form

$$
\begin{gathered}
\tilde{X}=\mu D+\hat{g} \quad \text { for } g=g_{1}, g_{4}, g_{5}, g_{6}, \\
\tilde{X}=e^{\lambda t+\omega \cdot x} \tilde{g} \quad \text { for } g=g_{1}, g_{2}, \\
\tilde{X}=e^{\lambda t} \hat{g} \quad \text { for any } g \text { Eq. (30). }
\end{gathered}
$$

Formulae (30), (31) give the principal description of all possible one-dimension algebras $\mathcal{A}$ which can be admitted by equation (2).

To describe two-dimension algebras $\mathcal{A}$ we classify matrices $g$ (27) forming twodimension Lie algebras. Up to equivalence transformations (25) there exist six such algebras: 


$$
\begin{gathered}
A_{2,1}=\left\{g_{3}, \tilde{g}_{2}\right\}, \quad A_{2,2}=\left\{g_{1}, g_{5}\right\}, \quad A_{2,3}=\left\{g_{5}, \tilde{g}_{2}\right\}, \quad A_{2,4}=\left\{g_{6}, \tilde{g}_{2}\right\}, \\
A_{2,5}=\left\{g_{1}, g_{2}\right\}, \quad A_{2,6}=\left\{g_{1}, g_{3}\right\},
\end{gathered}
$$

where $\tilde{g}_{2}$ is matrix $g_{2}$ (30) with $\lambda=0$.

Algebras (32) are Abelian while (33) are characterized by the following commutation relations:

$$
\left[e_{1}, e_{2}\right]=e_{2} \text {. }
$$

Two-dimension algebras $\mathcal{A}$ generated by (32) and (33) are spanned on the following basis elements:

$$
\begin{gathered}
\left\langle\mu D+\hat{e}_{1}+v t \hat{e}_{2}, \hat{e}_{2}\right\rangle, \quad\left\langle\mu D+\hat{e}_{2}+v t \hat{e}_{1}, \hat{e}_{1}\right\rangle, \\
\left\langle\mu D-\hat{e}_{1}, v D-\hat{e}_{2}\right\rangle, \quad\left\langle F_{1} \hat{e}_{1}+G_{1} \hat{e}_{2}, F_{2} \hat{e}_{1}+G_{2} \hat{e}_{2}\right\rangle
\end{gathered}
$$

and

$$
\left\langle\mu D-\hat{e}_{1}, \hat{e}_{2}\right\rangle, \quad\left\langle\mu D+\hat{e}_{1}+v t \hat{e}_{2}, \hat{e}_{2}\right\rangle
$$

respectively, where $\left\{F_{1}, G_{1}\right\}$ and $\left\{F_{2}, G_{2}\right\}$ are fundamental solutions of the following system:

$$
F_{t}=\lambda F+\alpha G, \quad G=\sigma F+\gamma G
$$

with arbitrary parameters $\lambda, \alpha, \sigma, \gamma$. Arbitrary parameters $\mu$ and $\nu$ in particular can be equal to zero.

In addition, there exist two dimension algebras $\mathcal{A}$ which are induced by onedimension algebras of matrices $g$ (27), namely

$$
\langle F \hat{g}, G \hat{g}\rangle, \quad\left\langle\mu D+\lambda e^{v t+\omega \cdot x} \hat{g}, e^{v t+\omega \cdot x} \hat{g}\right\rangle
$$

with $F$ and $G$ satisfying (37). Such algebras correspond to incompatible classifying equations (21).

Up to transformations (28) there exist four three-dimension algebras $A_{3,1}-A_{3,4}$ of matrices (27) and the only four-dimension algebra of such matrices which we denote as $A_{4}$ (Table 1).

Table 1. Three- and four-dimension algebras of matrices (27)

\begin{tabular}{c|cc}
\hline Algebra & Basis elements & Nonzero commutators \\
\hline$A_{3,1}$ & $e_{1}=g_{1}, e_{2}=g_{3}, e_{3}=\tilde{g}_{2}$ & {$\left[e_{1}, e_{2}\right]=e_{2},\left[e_{1}, e_{3}\right]=e_{3}$} \\
$A_{3,2}$ & $e_{1}=g_{5}, e_{2}=g_{1}, e_{3}=\tilde{g}_{2}$ & {$\left[e_{2}, e_{3}\right]=e_{3}$} \\
$A_{3,3}$ & $e_{1}=\tilde{g}_{2}, e_{2}=g_{5}, e_{3}=g_{6}$ & {$\left[e_{2}, e_{3}\right]=e_{1}$} \\
$A_{3,4}$ & $e_{1}=\tilde{g}_{2}, e_{2}=g_{3}, e_{3}=g_{4}$ & {$\left[e_{1}, e_{2}\right]=e_{2},\left[e_{1}, e_{3}\right]=e_{2}+e_{3}$} \\
$A_{4}$ & $e_{1}=g_{1}, e_{2}=g_{3}, e_{3}=\tilde{g}_{2}$ & {$\left[e_{1}, e_{2}\right]=e_{2},\left[e_{1}, e_{3}\right]=e_{3}$} \\
& $e_{4}=g_{5}$ & {$\left[e_{4}, e_{2}\right]=e_{3}$} \\
\hline
\end{tabular}

Using commutation relations present in the table we come to the following related three-dimension algebras $\mathcal{A}$ :

$$
\left\langle\mu D-\hat{e}_{1}, \hat{e}_{2}, \hat{e}_{3}\right\rangle, \quad\left\langle\hat{e}_{1}, F_{1} \hat{e}_{2}+G_{1} \hat{e}_{3}, F_{2} \hat{e}_{2}+G_{2} \hat{e}_{3}\right\rangle
$$


with $e_{a}$ belonging to $A_{3,1}$;

$$
\left\langle\mu D-2 \hat{e}_{1}, v D-2 \hat{e}_{2}, \hat{e}_{3}\right\rangle
$$

with $e_{a}$ belonging to $A_{3,2}$;

$$
\begin{gathered}
\left\langle\mu D-2 \hat{e}_{2}, v D-2 \hat{e}_{3}, \hat{e}_{1}\right\rangle, \quad\left\langle\hat{e}_{1}, D+2 e_{\alpha}+2 v t \hat{e}_{1}, \hat{e}_{\alpha^{\prime}}\right\rangle, \\
\left\langle e^{v t+\omega \cdot x} \hat{e}_{1}, e^{v t+\omega \cdot x} \hat{e}_{\alpha}, \hat{e}_{\alpha^{\prime}}\right\rangle,
\end{gathered}
$$

where $\alpha, \alpha^{\prime}=2,3, \alpha^{\prime} \neq \alpha$ and $e_{a}$ belong to $A_{3,3}$;

$$
\left\langle\mu D-2 \hat{e}_{1}, \hat{e}_{2}, \hat{e}_{3}\right\rangle, \quad\left\langle\hat{e}_{1}, e^{v t+\omega \cdot x} \hat{e}_{2}, e^{v t+\omega \cdot x} \hat{e}_{3}\right\rangle
$$

with $e_{a}$ belonging to $A_{3,4}$.

The four-dimensional algebra $A_{4}$ induces algebras $\mathcal{A}$ given below:

$$
\begin{aligned}
& \left\langle\mu D-2 \hat{e}_{1}, v D-2 \hat{e}_{4}, \hat{e}_{2}, \hat{e}_{3}\right\rangle, \\
& \left\langle e^{v t+\omega \cdot x} \hat{e}_{1}, e^{v t+\omega \cdot x} \hat{e}_{4}, \hat{e}_{2}, \hat{e}_{3}\right\rangle .
\end{aligned}
$$

Thus we had specified algebras of main symmetries which can be admitted by equation (2).

5. Solution of classifying equations for the case of invertible diffusion matrix. Applying results of the previous section we can easily classify main symmetries of equation (2). Such classification reduces to solving equations (21) with their known coefficients $C^{1}, C^{2}$ and $B^{1}, B^{2}$ which can be found comparing (20) with the found realizations of algebras $\mathcal{A}$. To complete the group classification of equations (2) we will specify all cases when relations (22), (23) are satisfied, i.e., when the main symmetries can be extended.

Solving of equations (21) with known $C^{1}, C^{2}$ and $B^{1}, B^{2}$ is a rather routine procedure. We restrict ourselves to presentation of an example of such solution in the following. Note that asking for invariance of (2) with respect to one-dimension algebra $\mathcal{A}$ we fix the nonlinearities $f^{1}$ and $f^{2}$ up to arbitrary functions while an invariance with respect to a two-dimension algebra $\mathcal{A}$ usually fixes these nonlinearities up to arbitrary parameters.

We will solve classifying equations (21) up to equivalence transformations $u \rightarrow$ $\rightarrow \tilde{u}=U(u, v, t, x), v \rightarrow \tilde{v}=V(u, v, t, x), t \rightarrow \tilde{t}=T(u, v, t, x), x \rightarrow \tilde{x}=X(u, v, t$, $x)$ and $f^{a} \rightarrow \tilde{f}^{a}=F^{a}\left(u, v, t, x, f^{a}\right)$ which keep the general form of equations (2) but can change functions $f^{1}$ and $f^{2}$.

The group of equivalence transformations for equation (2) can be found using the classical Lie approach and treating $f^{1}$ and $f^{2}$ as additional dependent variables. In accordance with their definition, equivalence transformations include all symmetry transformations (i.e., transformations generated by operators (19) and other symmetries which will be found in the following) and also transformations (25). In addition, for some particular nonlinearities $f^{1}$ and $f^{2}$ there exist additional equivalence transformations, whose list is given in formulae:

1. $u \rightarrow \exp (\omega t) u, \quad v \rightarrow \exp (\omega t) v$,

2. $u \rightarrow u+\omega t, \quad v \rightarrow v$,

3. $u \rightarrow u, \quad v \rightarrow v+\rho t$,

4. $u \rightarrow u, \quad v \rightarrow v+\rho t u$,

5. $u \rightarrow u, \quad v \rightarrow v-\rho t u+\rho \delta \frac{t^{2}}{2}$, 
6. $u \rightarrow e^{2 \rho t} u, \quad v \rightarrow e^{2 \rho t}\left(v+\omega t u+\rho t^{2} u\right)$,

7. $u \rightarrow u+\rho t, \quad v \rightarrow v+\rho t u+\rho^{2} \frac{t^{2}}{2}$,

8. $u \rightarrow e^{\kappa t} u, \quad v \rightarrow e^{\kappa t}(v-v \kappa \eta t u)$,

9. $u \rightarrow u+3 \omega t, \quad v \rightarrow v+3 \omega t^{2} u+6 \omega^{2} t^{3}+\rho t u+3 \omega \rho t u^{2}$,

10. $u \rightarrow e^{v \omega t} u, \quad v \rightarrow e^{v \omega t}(v-\sigma \omega t u)$,

11. $u \rightarrow e^{\lambda \omega t^{2}} u, \quad v \rightarrow e^{\lambda \omega t^{2}}(v+2 \omega t u)$,

12. $u \rightarrow u+\rho t, \quad v \rightarrow v+\eta \rho t$,

13. $u \rightarrow u, \quad v \rightarrow v+\rho x_{m}$.

In the following we specify additional equivalence transformations (39) admitted by some of equations (2).

Let us present an example of solving of classifying equations. Consider the first of algebras $\mathcal{A}$ given by relation (35) with $e_{1}, e_{2}$ belonging to algebra $A_{2,2}$ (32). It includes two basis elements

$$
X_{1}=\mu D-u \partial_{u}-v \partial_{v}, \quad X_{2}=v D-u \partial_{v} .
$$

Comparing $X_{1}$ with $\tilde{X}$ (20) we conclude that in this case $C^{1}=1, C^{2}=B^{1}=B^{2}=$ $=0$ and so the classifying equations (21) are reduced to the following ones:

$$
(\mu+1) f^{a}=\left(u \partial_{u}+v \partial_{v}\right) f^{a}, \quad a=1,2 .
$$

General solutions of this system have the form

$$
f^{1}=u^{\mu+1} F_{1}, \quad f^{2}=v^{\mu+1} F_{2}
$$

where $F_{1}$ and $F_{2}$ are arbitrary functions of $\frac{u}{v}$.

Asking for symmetry of equation (2) with respect to transformations generated by $X_{2}$ we come to the following classifying equations (21):

$$
\begin{gathered}
v f^{1}=u \partial_{v} f^{1}, \\
v f^{2}+f^{1}=u \partial_{v} f^{2},
\end{gathered}
$$

Substituting (40) into (41) we come to the equation whose general solution for $\mu \neq$ $\neq 0$ is

$$
\begin{gathered}
f^{1}=\lambda u^{\mu+1} e^{v v / u}, \\
f^{2}=e^{v v / u}(\lambda v+\sigma u) u^{\mu} .
\end{gathered}
$$

In the special case $\mu=0$ the solution has the form

$$
\begin{gathered}
f^{1}=\lambda u e^{v v / u}+\omega u, \\
f^{2}=e^{v v / u}(\lambda v+\sigma u)+\omega v .
\end{gathered}
$$


However, in this case there exist the additional equivalence transformation (39), Item 1 which reduces parameter $\omega$ in (43) to zero. So without loss of generality we can restrict ourselves to solutions (42) for any $\mu$.

Thus equation (2) admits the two-dimension algebra of main symmetries spanned on $X_{1}, X_{2}$ provided $f^{1}$ and $f^{2}$ have the form (42). This symmetry can be extended if functions (42) and $f^{2}$ satisfy one of conditions (22), (23) or both the conditions (22), (24).

Equation (23) is incompatible with (42). In order equation (22) be satisfied we have to impose the condition $\mu=-a v$ on parameters $\mu, v$ and $a$. The related equation (2), (42) has the form

$$
\begin{gathered}
u_{t}-a \Delta u=\lambda u^{1-a v} e^{v v / u} \\
u_{t}-\Delta u-a \Delta v=e^{v v / u}(\lambda v+\sigma u) u^{-a v}
\end{gathered}
$$

and admits the Galilei generators $G_{\mu}$ (12). Finally, asking for equation (24) be satisfied we obtain one more condition $v=-\frac{4}{a} m$ which guaranties invariance of equation (44) with respect to the conformal generator $K$ of (12).

The obtained classification results are presented in Table 2, Item 3.

In analogous way we solve classifying equations for other algebras $\mathcal{A}$ and specify the cases when the main symmetry can be extended.

6. Classification results for equations (2) with invertible diffusion matrix. The classification results are given in the following Tables $1-5$ when we also indicate the additional equivalence transformations (AET) (39) which are admitted by some particular equations (2). The symbols $D, \hat{G}_{v}, G_{v}$ and $K$ denote operators given by Eq. (12).

In the following tables $F_{1}, F_{2}$ and $F$ are arbitrary functions whose arguments are specified in the third column, $\Psi(x)$ is an arbitrary function of $x_{1}, x_{2}, \ldots, x_{m}$ and $\psi_{v}(x)$ is a solution of the linear heat equation $\left(\partial_{t}-a \Delta\right) \psi_{v}=v \psi_{v}$. In addition, we denote by $\Psi_{\mu}(x)$ a solution of the Laplace equation $\Delta \Psi_{\mu}(x)=\mu \Psi_{\mu}(x)$.

The letters $\varepsilon, \eta$ and $\delta$ denote parameters which can take the values $\varepsilon= \pm 1, v=$ $=0,1$ and $\delta=0, \pm 1$; the other Greek letters denote parameters which can take any (including zero) real values.

Table 2. Nonlinearities with arbitrary functions,

\begin{tabular}{|c|c|c|c|}
\hline No. & Nonlinear terms & $\begin{array}{l}\text { Arguments } \\
\text { of } F_{1}, F_{2}, F\end{array}$ & $\begin{array}{c}\text { Symmetries and AET } \\
\text { Eq. (39) }\end{array}$ \\
\hline 1 & $\begin{array}{c}f^{1}=\delta u+F_{1} \\
f^{2}=\delta u^{2}+F_{1} u+F_{2}\end{array}$ & $2 v-u^{2}$ & $\psi_{\delta}\left(u \partial_{v}+\partial_{u}\right)$ \\
\hline 2 & $\begin{array}{c}f^{1}=e^{\eta u} F_{1} \\
f^{2}=e^{\eta u}\left(F_{2}+F_{1} u\right)\end{array}$ & $2 v-u^{2}$ & $\begin{array}{c}\eta D-u \partial_{v}-\partial_{u} \\
{[\text { AET } 7 \text { if } \eta=0]}\end{array}$ \\
\hline 3 & $\begin{array}{c}f^{1}=F_{1} \\
f^{2}=F_{2}+\delta v\end{array}$ & $u$ & $\begin{array}{c}\psi_{\delta} \partial_{v} \\
{[\text { AET } 3 \text { if } \delta=0]}\end{array}$ \\
\hline
\end{tabular}
symmetries and AET for equations (2) 
Continuation of Table 2

\begin{tabular}{|c|c|c|c|}
\hline No. & Nonlinear terms & $\begin{array}{l}\text { Arguments } \\
\text { of } F_{1}, F_{2}\end{array}$ & Symmetries and AET \\
\hline 4 & $\begin{array}{c}f^{1}=\alpha u+\eta \\
f^{2}=\delta v+F, \alpha \eta=0\end{array}$ & $u$ & $\begin{array}{c}\psi_{\delta} \partial_{v}, e^{(\delta-\alpha) t}(u-\eta t) \partial_{v} \\
{[\text { AET } 3 \text { if } \delta=0} \\
\text { AET } 4 \text { if } \delta=\alpha, \eta=0]\end{array}$ \\
\hline 5 & $\begin{array}{c}f^{1}=u^{2} \\
f^{2}=(u+\delta) v+F\end{array}$ & $u$ & $\begin{array}{c}e^{\delta t} u \partial_{v} \\
e^{\delta t}\left(\partial_{v}+t u \partial_{v}\right)\end{array}$ \\
\hline 6 & $\begin{array}{c}f^{1}=u^{2}-1 \\
f^{2}=(u+v) v+F\end{array}$ & $u$ & $\begin{array}{r}e^{(v+1) t}\left(u \partial_{v}+\partial_{v}\right) \\
e^{(v-1) t}\left(u \partial_{v}-\partial_{v}\right)\end{array}$ \\
\hline 7 & $\begin{array}{c}f^{1}=u^{2}+1 \\
f^{2}=(u+v) v+F\end{array}$ & $u$ & $\begin{array}{l}e^{v t}\left(\cos t u \partial_{v}-\sin t \partial_{v}\right) \\
e^{v t}\left(\sin t u \partial_{v}+\cos t \partial_{v}\right)\end{array}$ \\
\hline 8 & $\begin{array}{c}f^{1}=e^{v / u} F_{1} u \\
f^{2}=e^{v / u}\left(F_{1} v+F_{2}\right)\end{array}$ & $u$ & $D-u \partial_{v}$ \\
\hline 9 & $f^{1}=e^{v} F_{1}, f^{2}=e^{v} F_{2}$ & $u$ & $D-\partial_{v}$ \\
\hline 10 & $f^{1}=e^{u} F_{1}, f^{2}=e^{u} F_{2}$ & $v$ & $D-\partial_{u}$ \\
\hline 11 & $\begin{array}{c}f^{1}=\delta u+F_{1} \\
f^{2}=-\eta u+F_{2}\end{array}$ & $v$ & $e^{(\delta+a \eta) t} \Psi_{\eta}(x) \partial_{u}$ \\
\hline 12 & $\begin{array}{l}f^{1}=u\left(F_{1}+\varepsilon \ln u\right) \\
f^{2}=v\left(F_{2}+\varepsilon \ln u\right)\end{array}$ & $\frac{u}{v}$ & $e^{\varepsilon t}\left(u \partial_{u}+v \partial_{v}\right)$ \\
\hline 13 & $\begin{array}{c}f^{1}=u F_{1}+\delta \eta v \\
f^{2}=\delta \frac{u}{v}(u+\eta v)+ \\
+u F_{2}+v F_{1}\end{array}$ & $u e^{-\eta v / u}$ & $\begin{array}{c}e^{\delta t}\left(u \partial_{v}+\eta\left(u \partial_{u}+v \partial_{v}\right)\right) \\
\& \hat{G}_{\alpha} \text { if } a=-\eta, \delta \eta \neq 0 \\
\text { or } G_{\alpha} \text { if } a=-\eta \neq 0, \delta=0\end{array}$ \\
\hline 14 & $\begin{array}{c}f^{1}=u^{\varepsilon+1} F_{1} \\
f^{2}=u^{\varepsilon}\left(F_{2} u-F_{1} v\right)\end{array}$ & $u e^{v / u}$ & $\varepsilon D+u \partial_{v}-u \partial_{u}-v \partial_{v}$ \\
\hline 15 & $\begin{array}{l}f^{1}=u^{\mu+1} F_{1} \\
f^{2}=u^{\mu+1} F_{2}\end{array}$ & $\frac{v}{u}$ & $\begin{array}{c}\mu D-u \partial_{u}-v \partial_{v} \\
{[\mathrm{AET} 1 \text { if } \mu=0]}\end{array}$ \\
\hline
\end{tabular}


Table 3. Nonlinearities with arbitrary parameters and symmetries for equations (2) with $a \neq 0$

\begin{tabular}{|c|c|c|c|}
\hline No. & Nonlinear terms & Symmetries & AET (39) \\
\hline 1 & $\begin{array}{c}f^{1}=\delta, f^{2}=u^{v} \\
\delta=0 \text { if } v=2\end{array}$ & $\begin{array}{c}\psi_{0} \partial_{v},(u-\delta t) \partial_{v} \text { for any } v, \delta \\
\& u \partial_{u}+v v \partial_{v} \text { for } \delta=0 \\
\& \partial_{u}+2 t u \partial_{v} \text { for } v=2\end{array}$ & $\begin{array}{c}3,5 \& 9 \\
\text { if } \delta=0 \\
v=2\end{array}$ \\
\hline 2 & $\begin{array}{c}f^{1}=u, f^{2}=\varepsilon u^{v} \\
v \neq 0,1\end{array}$ & $\begin{array}{c}\psi_{0} \partial_{v}, e^{-t} u \partial_{v} \text { for any } v \\
\& e^{t}\left(u \partial_{v}+\varepsilon \partial_{u}\right) \text { for } v=2\end{array}$ & 3 \\
\hline 3 & $\begin{aligned} f^{1} & =\delta e^{u} \\
f^{2} & =\eta e^{u}\end{aligned}$ & $\begin{array}{c}D-\partial_{u}, \psi_{0} \partial_{v} \\
\& u \partial_{v} \text { if } \delta=0\end{array}$ & $\begin{array}{c}3 \& 4 \text { if } \\
\delta=0\end{array}$ \\
\hline 4 & $\begin{array}{c}f^{1}=\delta u^{v+1} e^{\eta v / u} \\
f^{2}=e^{\eta v / u}(\delta v+\sigma u) u^{v} \\
(v-1)^{2}+\eta+\sigma^{2} \neq 0\end{array}$ & $\begin{array}{c}\eta D-u \partial_{v}, v D-u \partial_{u}-v \partial_{v} \\
\& G_{\alpha} \text { if } v=\eta a \\
\& K \text { if } v=\frac{4}{m}\end{array}$ & $\begin{array}{c}8 \& 4 \text { if } \\
\eta=0\end{array}$ \\
\hline 5 & $f^{1}=u^{2}, f^{2}=u v$ & $D-u \partial_{u}-v \partial_{v}, u \partial_{v},(1+t u) \partial_{v}$ & 4 \\
\hline 6 & $f^{1}=\varepsilon u^{\mu+1}, f^{2}=0$ & $\mu D-u \partial_{u}-v \partial_{v}, \psi_{0} \partial_{v}$ & 3 \\
\hline 7 & $f^{1}=\eta v^{v+1}, f^{2}=\delta v^{v+1}$ & $v D-u \partial_{u}-v \partial_{v}, \Psi_{0}(x) \partial_{u}$ & 2 \\
\hline 8 & $f^{1}=\eta e^{v}, f^{2}=\delta e^{v}$ & $D-\partial_{v}, \Psi_{0}(x) \partial_{u}$ & 2 \\
\hline 9 & $f^{1}=\delta e^{u}, f^{2}=\varepsilon u e^{u}$ & $\begin{array}{c}\delta\left(D-\partial_{u}\right)-u \partial_{v}, \psi_{0} \partial_{v} \\
\& u \partial_{v} \text { if } \delta=0\end{array}$ & $\begin{array}{c}3 \& 4 \text { if } \\
\delta=0\end{array}$ \\
\hline 10 & $\begin{array}{c}f^{1}=\eta e^{2 v-u^{2}} \\
f^{1}=(\eta u+\mu) e^{2 v-u^{2}}\end{array}$ & $2 D-\partial_{v}, \partial_{v}+u \partial_{v}$ & 7 \\
\hline 11 & $\begin{array}{l}f^{1}=\eta \ln v \\
f^{2}=\delta \ln v\end{array}$ & $\begin{array}{l}\Psi_{0}(x) \partial_{u}, D+u \partial_{u}+v \partial_{v}+ \\
+\left((\eta-\delta a) t-\frac{\delta}{2 m} x^{2}\right) \partial_{u}\end{array}$ & 2 \\
\hline 12 & $\begin{array}{c}f^{1}=\delta \\
f^{2}=\varepsilon \ln u\end{array}$ & $\begin{array}{c}D+u \partial_{u}+v \partial_{v}+\varepsilon t \partial_{v} \\
\psi_{0} \partial_{v},(u-\delta t) \partial_{v}\end{array}$ & $\begin{array}{l}3,5 \& 4 \\
\text { if } \delta=0\end{array}$ \\
\hline
\end{tabular}


Continuation of Table 3

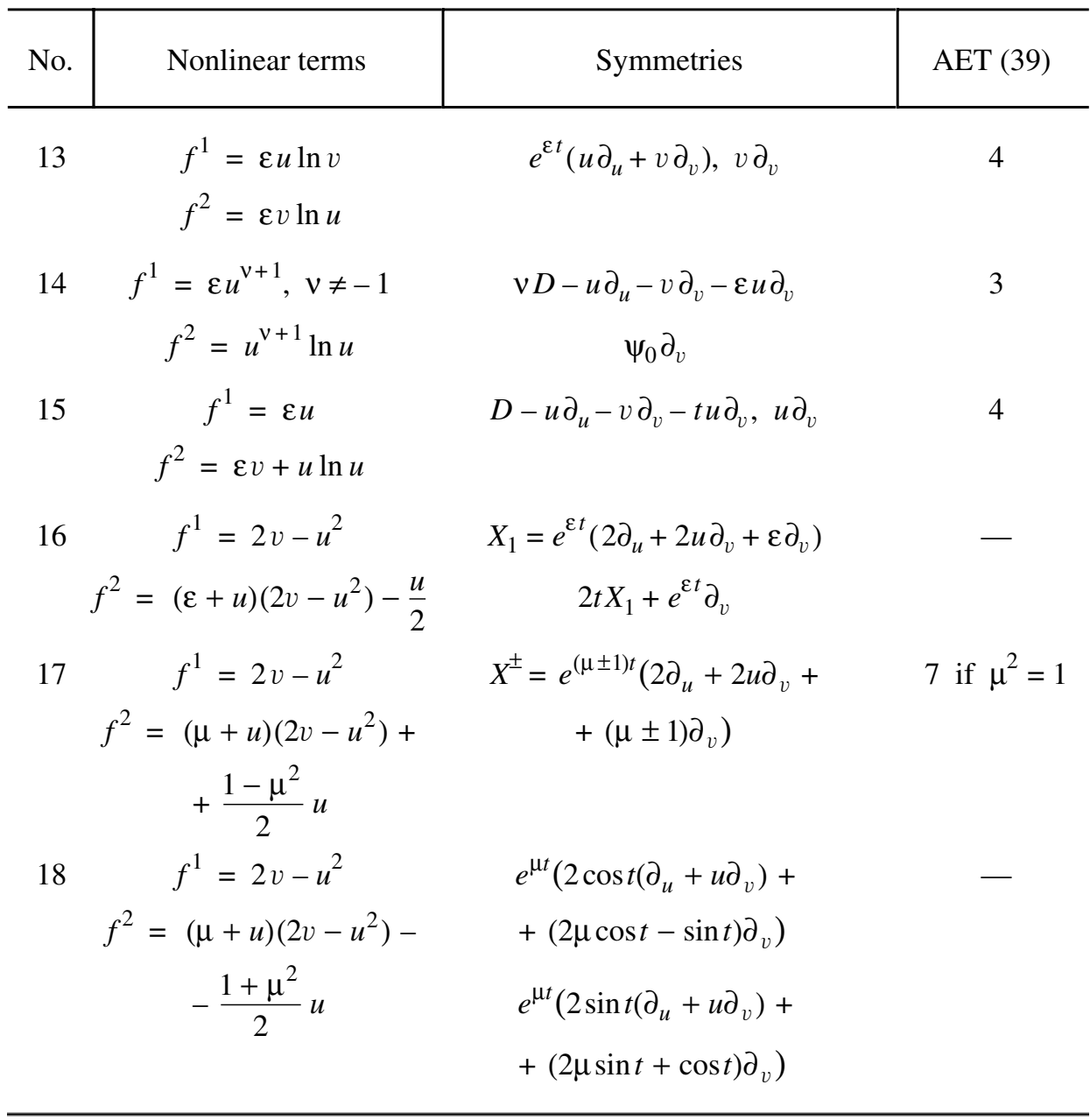

In the following table we present symmetries of a special subclass of equations (2) whose right-hand side is given in the table title. The following notations are used here: $\Delta=\frac{1}{4}(\mu-v)^{2}+\lambda \sigma, \Omega=\frac{1}{2}(\mu+v), \omega_{ \pm}=\Omega \pm 1$ where $\mu, v, \lambda$ and $\sigma$ are parameter used in definition of the related nonlinearities $f^{1}, f^{2}$.

Table 4. Symmetries of equations (2) with nonlinearities $f^{1}=\lambda v+\mu u \ln u, f^{2}=\lambda \frac{v^{2}}{u}+(\sigma u+\mu v) \ln u+v v$ and $a \neq 0$

\begin{tabular}{c|c|c|c}
\hline No. & $\begin{array}{c}\text { Conditions for } \\
\text { coefficients }\end{array}$ & $\begin{array}{c}\text { Symmetries } \\
\text { and AET Eq. (39) }\end{array}$ & Additional symmetries \\
\hline 1 & $\lambda=0$ & $e^{\delta t} u \partial_{v}, e^{\delta t}\left(R \partial_{R}+\sigma t u \partial_{v}\right)$ & $G_{\alpha}$ if $\sigma=\delta=0$ \\
& $\mu=v=\delta$ & [AET 6 if $\mu=0]$ & $\hat{G}_{\alpha}$ if $\sigma=0, \delta \neq 0$ \\
& & $\tilde{\psi}_{0} \partial_{v}$ if $\mu=0$ \\
\hline
\end{tabular}


Continuation of Table 4

\begin{tabular}{|c|c|c|c|}
\hline No. & $\begin{array}{l}\text { Conditions for } \\
\text { coefficients }\end{array}$ & $\begin{array}{c}\text { Symmetries } \\
\text { and AET Eq. (39) }\end{array}$ & Additional symmetries \\
\hline 2 & $\begin{array}{l}\lambda=0, \mu \neq v \\
\mu^{2}+v^{2}=1\end{array}$ & $\begin{array}{c}e^{\mu t}\left((\mu-v) R \partial_{R}+\sigma u \partial_{v}\right), e^{v t} u \partial_{v} \\
{[\text { AET } 10 \text { if } \mu \nu=0]}\end{array}$ & $\begin{array}{c}\hat{G}_{\alpha} \text { if } \mu \neq 0 \\
\sigma=a(v-\mu) \\
\psi_{v} \partial_{v} \text { if } \mu=0 \\
\& G_{\alpha} \text { if } a v=\sigma\end{array}$ \\
\hline 3 & $\begin{array}{c}\Delta=0 \\
\lambda=\varepsilon \\
\mu+\nu=2 \Omega\end{array}$ & $\begin{array}{c}X^{4}=e^{\Omega t}\left(2 \varepsilon R \partial_{R}+(\nu-\mu) u \partial_{v}\right) \\
2 e^{\Omega t} u \partial_{v}+t X^{4}[\text { AET } 10 \text { if } \\
\mu=-v \& 1,11 \text { if } \\
\mu=v=0]\end{array}$ & $\begin{array}{c}G_{\alpha} \text { if } \Omega=0 \\
v=-a \varepsilon \neq 0 \\
\hat{G}_{\alpha} \text { if } \Omega \neq 0 \\
2 a \varepsilon=(\mu-v) \neq 0\end{array}$ \\
\hline 4 & $\begin{array}{c}\lambda \neq 0 \\
\Delta=1 \\
\omega_{ \pm}=\Omega \pm 1\end{array}$ & $\begin{array}{c}e^{\omega_{+} t}\left(\lambda R \partial_{R}+\left(\omega_{+}-\mu\right) u \partial_{v}\right) \\
e^{\omega_{-} t}\left(\lambda R \partial_{R}+\left(\omega_{-}-\mu\right) u \partial_{v}\right) \\
{[\text { AET } 10 \text { if } \mu \nu=\lambda \sigma} \\
\& 1 \text { if } \mu=\sigma=0]\end{array}$ & $\begin{array}{c}G_{\alpha} \text { if } \mu=a \lambda \\
\omega_{+} \omega_{-}=0 \\
\hat{G}_{\alpha} \text { if } \omega_{+} \neq 0 \\
a \lambda=-\left(\omega_{+}-\mu\right)\end{array}$ \\
\hline 5 & $\Delta=-1$ & $\begin{array}{c}e^{\Omega t}\left[2 \lambda \cos t R \partial_{R}+\right. \\
\left.+((v-\mu) \cos t-2 \sin t) u \partial_{v}\right] \\
e^{\Omega t}\left[2 \lambda \sin t R \partial_{R}+\right. \\
\left.+((v-\mu) \sin t+2 \cos t) u \partial_{v}\right]\end{array}$ & none \\
\hline
\end{tabular}

7. Group classification of reaction-diffusion equations with nilpotent diffusion matrix. Consider now equations (3), (4) and specify their Lie symmetries. We restrict ourselves to the case $p \neq 0$ when generators of admitted Lie group have the general form (14) while the related classifying equations are given by formula (16). Moreover without loss of generality we put $p=1$.

The results of group classifications are presented in Table 5. All the corresponding equations (3) admit the additional equivalence transformations

$$
t \rightarrow \rho t, \quad x \rightarrow \rho^{2 / 3} x, \quad v \rightarrow \omega v, \quad u \rightarrow \omega \rho^{1 / 3} u .
$$

Table 5. Nonlinearities and symmetries for equations (3), (4) with $p=1$

\begin{tabular}{c|c|c|c}
\hline No. & Nonlinearities & $\begin{array}{c}\text { Arguments } \\
\text { of } F_{1}, F_{2}\end{array}$ & Symmetries \\
\hline 1 & $f^{1}=u^{1+3 \mu} F_{1}$ & $v u^{-\mu-1}$ & $2 \mu \tilde{D}-u \partial_{u}-v \partial_{v}$ \\
$f^{2}=u^{1+4 \mu} F_{2}$ & & [AET 1 if $\mu=0]$ \\
2 & $f^{1}=v^{3} F_{1}$ & $u-\eta \ln v$ & $\tilde{D}-\eta \partial_{u}$ \\
& $f^{2}=v^{4} F_{2}$ & & \\
\hline
\end{tabular}


Continuation of Table 5

\begin{tabular}{|c|c|c|c|}
\hline No. & Nonlinearities & $\begin{array}{l}\text { Arguments } \\
\text { of } F_{1}, F_{2}\end{array}$ & Symmetries \\
\hline 3 & $f^{1}=u\left(F_{1}+\eta \ln u\right)$ & $\frac{v}{u}$ & $e^{\eta t}\left(u \partial_{u}+v \partial_{v}\right)$ \\
\hline & $f^{2}=v\left(F_{2}+\eta \ln u\right)$ & & [AET 1 if $\eta=0$ ] \\
\hline 4 & $\begin{array}{l}f^{1}=u^{-2} F_{1} \\
f^{2}=u^{-3} F_{2}\end{array}$ & $v-\eta \ln u$ & $\tilde{D}+u \partial_{u}+v \partial_{v}+\eta \partial_{v}$ \\
\hline 5 & $\begin{array}{l}f^{1}=v v+F_{1} \\
f^{2}=\eta v+F_{2}\end{array}$ & $u$ & $\begin{array}{c}e^{\eta t-v x_{m}} \Psi(\tilde{x}) \partial_{v} \\
{[\text { AET 3,13 if } \eta=v=0]}\end{array}$ \\
\hline 6 & $\begin{array}{l}f^{1}=\tilde{\eta} u+F_{1} \\
f^{2}=\lambda u+F_{2}\end{array}$ & $\eta u-v$ & $\begin{array}{c}e^{\lambda t} \Psi_{\mu}\left(\tilde{x}, x_{m}+\tilde{\eta} t\right)\left(\partial_{u}+\eta \partial_{v}\right) \\
\mu=\tilde{\eta} \eta-\lambda[\text { AET } 12 \\
\quad \text { if } \lambda=\tilde{\eta}=0]\end{array}$ \\
\hline 7 & $\begin{array}{l}f^{1}=\mu u^{-2} v^{3} \\
f^{2}=v u^{-3} v^{4}\end{array}$ & & $\tilde{D}, u \partial_{u}+v \partial_{v}[$ AET 1] \\
\hline 8 & $\begin{array}{l}f^{1}=\eta e^{3 u} \\
f^{2}=v e^{4 u}\end{array}$ & & $\tilde{D}-\partial_{u}, \Psi(\tilde{x}) \partial_{v} \quad[$ AET 3] \\
\hline 9 & $\begin{array}{l}f^{1}=\alpha e^{-2 v} \\
f^{2}=\eta e^{-3 v}\end{array}$ & & $\begin{array}{c}\tilde{D}+u \partial_{u}+v \partial_{v}+\partial_{v}, \Psi_{0}(x) \partial_{u} \\
{[\text { AET 2] }}\end{array}$ \\
\hline 10 & $\begin{array}{l}f^{1}=\eta u^{3 \mu+1} \\
f^{2}=v u^{4 \mu+1}\end{array}$ & & $\begin{array}{c}\mu \tilde{D}-u \partial_{u}-v \partial_{v}, \Psi(\tilde{x}) \partial_{v} \\
{[\text { AET 3, 13] }}\end{array}$ \\
\hline 11 & $\begin{array}{l}f^{1}=\alpha v^{2 v+1} \\
f^{2}=\eta v^{3 v+1}\end{array}$ & & $\begin{array}{c}v \tilde{D}-u \partial_{u}-v \partial_{v}, \Psi_{0}(x) \partial_{u} \\
{[\text { AET 2] }}\end{array}$ \\
\hline 12 & $\begin{aligned} f^{1} & =\alpha u^{1 / 4} \\
f^{2} & =\delta \ln u\end{aligned}$ & & $\begin{array}{c}\frac{1}{4} \tilde{D}+v \partial_{v}+u \partial_{u}+\delta t \partial_{v}, \Psi(\tilde{x}) \partial_{v} \\
{[\text { AET 3, 13] }}\end{array}$ \\
\hline 13 & $\begin{aligned} f^{1} & =v \ln v \\
f^{2} & =\frac{\eta}{\sqrt{v}}\end{aligned}$ & & $\begin{array}{c}\frac{1}{3} \tilde{D}+u \partial_{u}+v \partial_{v}+\eta u t \partial_{u}, \Psi_{0}(x) \partial_{u} \\
{[\text { AET 2] }}\end{array}$ \\
\hline
\end{tabular}

Here $\Psi_{\mu}(x)$ and $\Psi\left(\tilde{x}, x_{m}+t\right)$ are arbitrary solutions of the Laplace equation $\Delta \Psi_{\mu}=\mu \Psi_{\mu}$ in $m$-dimensional space, $\tilde{\Psi}_{\mu}(\tilde{x})$ is a solution of the Laplace equation in $(m-1)$-dimensional space, $\tilde{x}=\left(x_{1}, x_{2}, \ldots, x_{m-1}\right)$. Finally, we denote $\tilde{D}=3 t \partial_{t}+$ $+2 x_{v} \partial_{v}-v \partial_{v}$.

We solve the classifying equations using the technique developed in Sections 5 and 6. The general analysis of admissible algebras $\mathcal{A}$ can be carried out in complete 
analogy with Section 4. Moreover, the results present in Section 4 can be extended to the case of equations (3), (4) provided we make a formal change $D \rightarrow \tilde{D}=3 t \partial_{t}+$ $+2 x_{v} \partial_{v}-v \partial_{v}$ in all formulae where the operator $D$ appeared, and exclude all algebras $\mathcal{A}$ where matrices $g_{4}, g_{5}$ and $g_{6}$ (30) appear. Of course it is necessary to take into account that in contrast with $D$ operator $\tilde{D}$ does not commute with $\partial_{v}$. As a result we come to the following one-dimension algebras

$$
\begin{gathered}
\tilde{X}_{1}^{(1)}=\mu \tilde{D}-u \partial_{u}-v \partial_{v}, \\
\tilde{X}_{1}^{(2)}=\tilde{D}-v \partial_{u}, \quad \tilde{X}_{2}^{(v)}=e^{v t}\left(u \partial_{u}+v \partial_{v}\right), \\
\tilde{X}_{1}^{(3)}=\tilde{D}+u \partial_{u}+v \partial_{v}+v \partial_{v}, \quad \tilde{X}_{3}^{(3)}=e^{\sigma_{3} t+\rho_{3} x}\left(\partial_{u}+\partial_{v}\right), \\
\tilde{X}_{3}^{(1)}=e^{\sigma_{3} t+\rho_{1} \cdot x} \partial_{u}, \quad \tilde{X}_{3}^{(2)}=e^{\sigma_{2} t+\rho_{2} \cdot x} \partial_{v}
\end{gathered}
$$

and two-dimension algebras

$$
\begin{array}{ll}
\tilde{A}_{1}=\left\langle\tilde{D}, \tilde{X}_{2}^{(0)}\right\rangle, \quad \tilde{A}_{2}=\left\langle\tilde{X}_{1}^{(2)}, \tilde{X}_{3}^{(3)}\right\rangle, \quad \tilde{A}_{3}=\left\langle\tilde{X}_{1}^{(3)}, \tilde{X}_{3}^{(1)}\right\rangle, \\
\tilde{A}_{4}=\left\langle\tilde{X}_{1}^{(1)}, \tilde{X}_{3}^{(1)}\right\rangle, & \tilde{A}_{6}=\left\langle\tilde{D}+4\left(u \partial_{u}+v \partial_{v}\right)+t \partial_{v}, \tilde{X}_{3}^{(2)}\right\rangle, \\
\tilde{A}_{5}=\left\langle\tilde{X}_{1}^{(1)}, \tilde{X}_{3}^{(2)}\right\rangle, & \tilde{A}_{7}=\left\langle\tilde{D}+3\left(u \partial_{u}+v \partial_{v}\right)+t \partial_{v}, \tilde{X}_{3}^{(1)}\right\rangle .
\end{array}
$$

In this way the problem of group classification of the equations with the first order derivative terms reduces to solving the classifying equations (16) which their known coefficients $B^{1}, B^{2}, F$ and specifying the case when these equations have nontrivial solutions. Solving the related classifying equations (16) we easily find the related nonlinearities $f^{1}, f^{2}$ which are given in Table 5 .

In accordance with the results present in Table 7 equations (3), (4) with $p \neq 0$ can admit neither Galilei nor conformal symmetry transformations. This result follows directly from formulae (14), (15).

8. Discussion. We complete the group classification of systems of reactiondiffusion equations started in papers $[1,2]$, where systems with a diagonal and square diffusion matrix are studied. To save a room we do not present the classification results for equations (3) with $p=0$ which can be found in preprint [3].

The case of triangular diffusion matrix considered in the present paper appears to be rather complicated due to very large number of versions with different symmetries.

In Table 2 equations defined up to arbitrary functions are presented.

Tables 3 and 4 include the results of classification of equations (2) which are defined up to arbitrary parameters.

Note that we did not consider linear equations whose group classification is a rather trivial problem.

Among the classified equations there are only seven of them being invariant with respect to the Galilei transformations. The general form of Galilei-invariant equations (2) is

$$
\begin{aligned}
u_{t}-a \Delta u & =u F_{1}, \\
v_{t}-\Delta u-a \Delta v & =u F_{2}-v F_{1},
\end{aligned}
$$

where $F_{1}$ and $F_{2}$ are arbitrary functions of variable $\xi=u e^{v / u}$.

The only system of equations (2) which admits the extended Galilei group including the dilatation and conformal transformations is given by formula (44). 
Thus we have completed the group classification of systems of coupled reactiondiffusion equations (1) started in paper [4] and continued with varying success in [5 $10]$ and $[1,2]$. The number of nonequivalent equations of this type appears to be very large $(>300)$. Nevertheless, using the approach presented in [1] it was possible to make an effective classification of pairs of coupled reaction-diffusion equations with general diffusion matrix. Moreover, we classify the equations with arbitrary number of independent variables.

A survey of results of group classification of systems of reaction-diffusion equations with general diffusion matrix can be found in [11]. Essentially new point of the present paper (in comparison with [11] is completed description of equivalence transformations and their systematical use to simplify the classified equations.

We notice that group analysis of reaction-diffusion equations is being intensively developed in many lines including equations with arbitrary elements depending on $t, x$, $u, u_{t}, u_{x}, \ldots$, refer to [12] for a survey. Exact solutions generated by conditional symmetry of multidimensional diffusion equations where found in paper [13]. Thus our analysis of systems of diffusion equations is nothing but a part of general study of diffusion models which is currently rather popular.

1. Nikitin A. G. Group classification of systems of non-linear reaction-diffusion equations with general diffusion matrix. I. Generalized Ginzburg - Landau equations // J. Math. Anal. and Appl. 2006. - 324, Issue 1. - P. $615-628$.

2. Nikitin A. G. Group classification of systems of non-linear reaction-diffusion equations with general diffusion matrix. II. Generalized Turing systems // J. Math. Anal. and Appl. - 2007, doi:10.1016/j.jmaa.2006.10.032.

3. Nikitin A. G. Group classification of systems of non-linear reaction-diffusion equations with general diffusion matrix. III. Triangular diffusion matrix / math-ph/0502048.

4. Danilov $Y$ u. A. Group analysis of the Turing systems and of its analogues. - 1980. - (Preprint / Kurchatov Inst. Atomic Energy, IAE-3287/1).

5. Nikitin A. G., Wiltshire R. Symmetries of systems of nonlinear reaction-diffusion equations // Proc. Inst. Math. Nat. Acad. Sci. Ukraine. - 2000 - 30. - P. 47 - 59.

6. Cherniha R. M., King J. R. Lie symmetries of nonlinear multidimensional reaction-diffusion systems. I // J. Phys. A: Math. and Gen. - 2000. - 33. - P. $267-282$.

7. Cherniha R. M., King J. R. Lie symmetries of nonlinear multidimensional reaction-diffusion systems. I. Addendum // Ibid. - P. 7839 - 7841.

8. Nikitin A. G., Wiltshire R. Systems of reaction diffusion equations and their symmetry properties // J. Math. Phys. - 2001. - 42. - P. 1667

9. Nikitin A. G., Popovych R. O. Group classification of nonlinear Schrödinger equations // Ukr. Mat. Zh. $-2001 .-53$, № 8. - P. $1053-1060$.

10. Cherniha R. M., King J. R. Lie symmetries of nonlinear multidimensional reaction-diffusion systems. II // J. Phys. A: Math. and Gen. - 2002. - 36. - P. 405 - 425.

11. Nikitin A. G. Group classification of systems of nonlinear reaction-diffusion equations // Ukr. Math. Bull. - 2005. - 2, № 2. - P. 153 - 204.

12. Popovych R. O., Ivanova N. M. New results on group classification of nonlinear diffusionconvection equations // J. Phys. A: Math. and Gen. - 2004. - 37, № 30. - P. 7547 - 7565.

13. Barannyk T. A. Conditional symmetries and exact solutions for multidimensional diffusion equation // Ukr. Mat. Zh. - 2002. - 54, № 10. - P. 1416 - 1460.

Received 02.10.2006 